\title{
Die Geschichte des Sauerstoffs - von der Entdeckung bis zur medizinischen Anwendung
}

\author{
The History of Oxygen - From its Discovery to its Implementation as Medical \\ Therapy
}

Autoren

Institute
N. Koehler, M. Goubeaud, O. Hildebrandt, A. K. Sohrabi, U. Koehler

Klinik für Innere Medizin, SP Pneumologie, Intensiv- und Schlafmedizin (Direktor: Prof. Dr. C. Vogelmeier) Philipps-Universität Marburg

\section{Bibliografie}

DOI http://dx.doi.org/

10.1055/s-0030-1256779

Online-Publikation: 7. 9. 2011

Pneumologie 2011; 65:

736-741 @ Georg Thieme

Verlag KG Stuttgart · New York ISSN 0934-8387

\section{Korrespondenzadresse} Prof. Dr. Ulrich Koehler Klinik für Innere Medizin SP Pneumologie, Intensiv- und Schlafmedizin

Universitätsklinikum Gießen und Marburg $\mathrm{GmbH}$ Baldingerstraße 1 35033 Marburg koehleru@staff.uni-marburg.de

\section{Sauerstoff - biologische Bedeutung und Verwendung}

Sauerstoff ist ein farbloses Gas, das weder Geruch noch Geschmack hat. Erst bei einer Temperatur unter $-183^{\circ} \mathrm{C}$ verflüssigt es sich, bei $-218,4^{\circ} \mathrm{C}$ erstarrt es zu Kristallen. Sauerstoff ist für alle biologischen Organismen essentiell und eine Grundvoraussetzung für den Atmungsvorgang und den Energiemetabolismus. Wenn der Mensch in Ruhe ist, so verbraucht er etwa 200 bis 300 ml Sauerstoff in der Minute. Bei schwerer Arbeit oder sportlicher Betätigung benötigt er bis zu zehnmal so viel wie in Ruhe. Da der Mensch keine ausreichenden Speicherkapazitäten für Sauerstoff hat, muss ständig eine ausreichende Zufuhr gewährleistet sein. Die Sauerstofftherapie ist für Patienten mit chronischen Lungenerkrankungen wie COPD, Lungenemphysem und Lungenfibrose eine wichtige Voraussetzung zur Erhaltung der körperlichen Mobilität. Sauerstoff kann dem Patienten sowohl über Flüssigsauerstoffsysteme als auch Sauerstoffkonzentratoren appliziert werden. Mobile sauerstoffbedürftige Patienten werden in der Regel mit Flüssigsauerstoffsystemen versorgt. Von einem großen Vorratsbehälter, in dem der Sauerstoff auf -183 Grad tiefgekühlt ist, können kleinere tragbare Einheiten abgefüllt werden. Medizinisch verflüssigter Sauerstoff ermöglicht die Deposition größerer Mengen im Vergleich zu Sauerstoff in Gasform. Ein Liter Flüssigsauerstoff ergibt ca. 850 Liter gasförmigen Sauerstoff. Ein stationärer Behälter mit einem Fassungsvermögen von etwa 40 Litern Sauerstoff kann einen Patienten mit 34000 Litern Sauerstoff in Gasform versorgen. In Abhängigkeit der Flussrate ist diese Menge ausreichend für etwa ein bis zwei Wochen.

\section{Die Entdeckung des Sauerstoffs \\ $\nabla$}

Von der Entdeckung des Sauerstoffs bis hin zur Verflüssigung und der industriellen und medizinischen Nutzung war es ein langer Weg. Die Entdeckung des Sauerstoffs sowie die Beschreibung erster grundlegender physiologischer und physikalisch-chemischer Abläufe zu Atmung und Gasaustausch erfolgten im 18. Jahrhundert. Henry Cavendish (1731-1810) isolierte 1766 erstmals Wasserstoff aus der Luft, Daniel Rutherford (1749-1819) wenige Jahre später Stickstoff. Sauerstoff wurde von dem schwedisch-deutschen Apotheker Carl Wilhelm Scheele (1742-1786) und dem englischen Chemiker Joseph Priestley (1733 - 1804) in den 70er-Jahren des 18. Jahrhunderts unabhängig voneinander entdeckt. Scheele

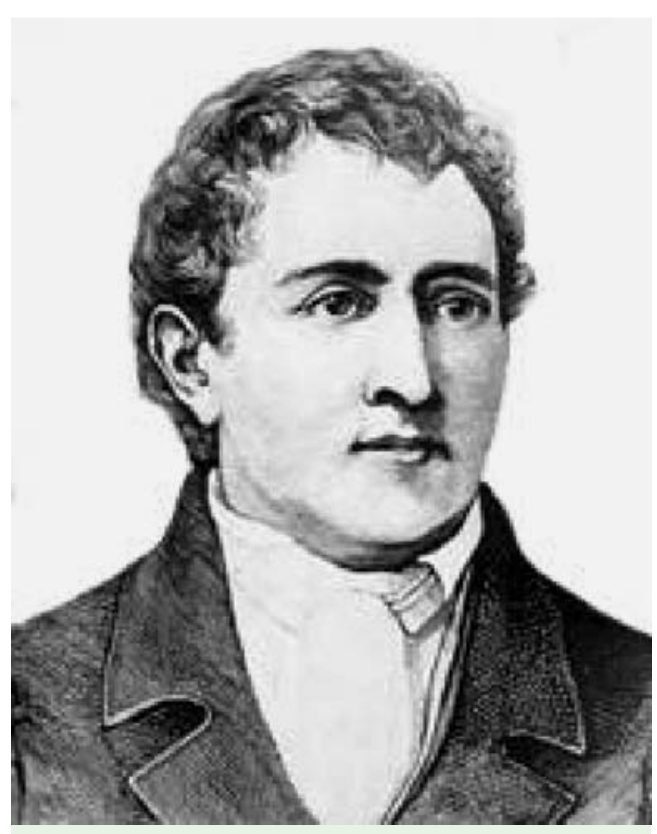

Abb. 1 Carl Wilhelm Scheele (1742-1786). Quelle: Wikipedia. 


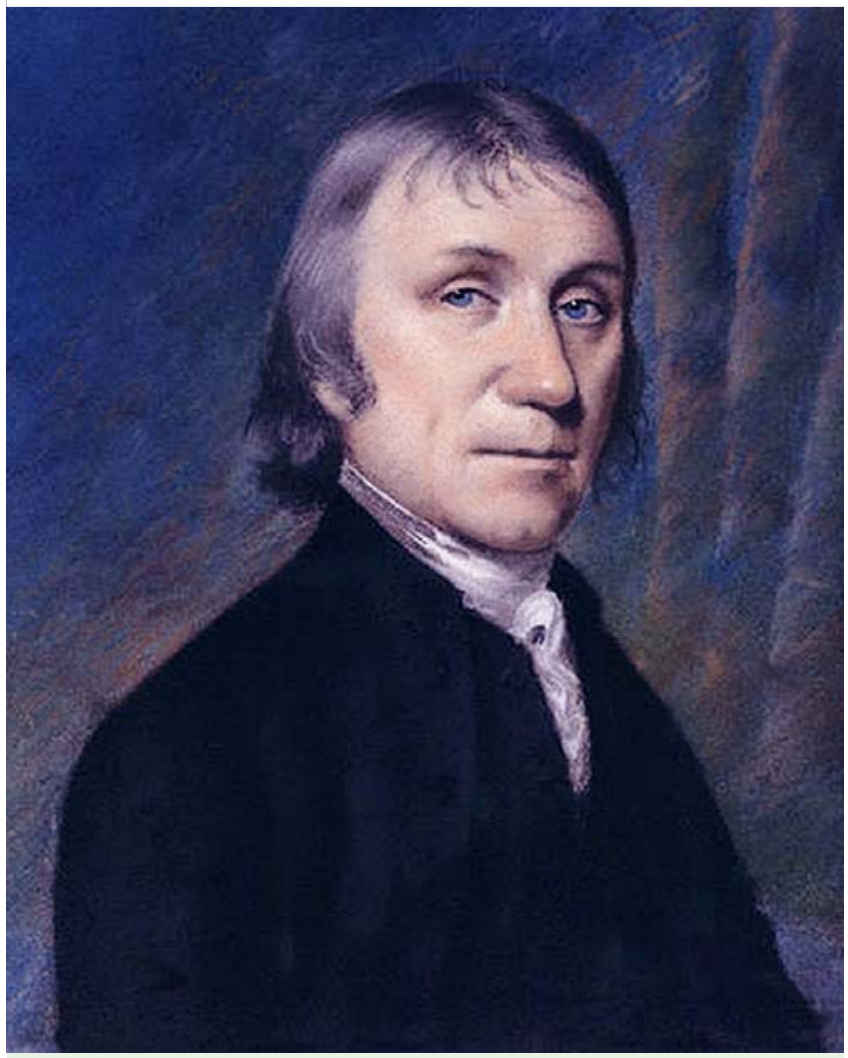

Abb. 2 Joseph Priestley (1733-1804). Quelle: Wikimedia Commons.

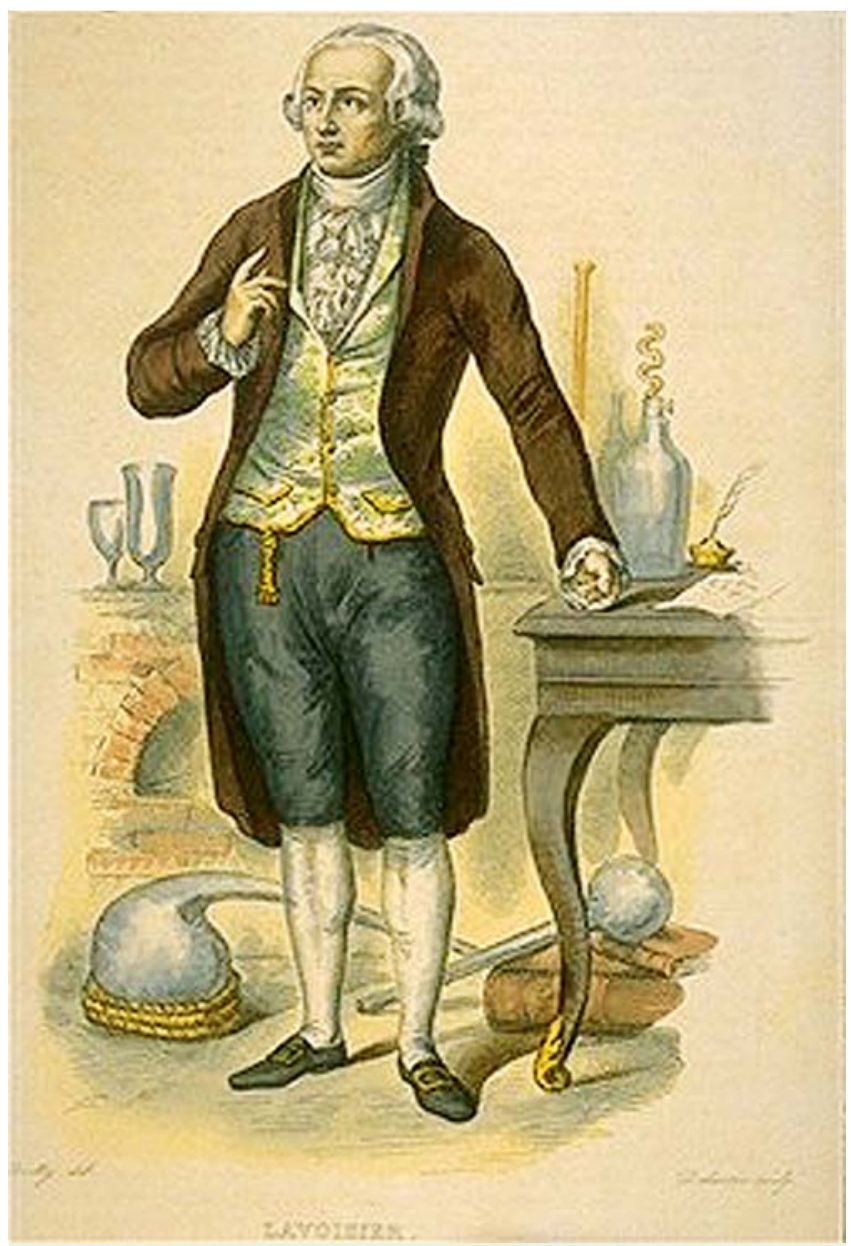

Abb. 3 Antoine Lavoisier (1743-1794). Quelle: Wikimedia Commons. erhitzte Braunstein $\left(\mathrm{MnO}_{2}\right)$ mit konzentrierter Schwefelsäure und beobachtete bei dieser Reaktion das Aufsteigen eines Gases, das er „Vitriol- bzw. Feuerluft“ nannte. Joseph Priestley experimentierte mit Quecksilberoxid und beobachtete ebenfalls eine mysteriöse Gasentwicklung. Priestley publizierte seine Ergebnisse 1775 , Scheeles Veröffentlichung erschien 1777. Weder Scheele noch Priestley erkannten jedoch die historische Bedeutung ihrer Entdeckung. Erst durch den Franzosen Antoine Lavoisier (17431794) wurde Sauerstoff als essentieller Bestandteil der Luft identifiziert. Lavoisier verdanken wir die Erkenntnis, dass Sauerstoff, der mit der Atmung aus der Luft aufgenommen wird, die Lebensvorgänge von Mensch und Tier unterhält. 1778 beschrieb Lavoisier die Atmung als einen aus zwei Abläufen bestehenden Mechanismus, dem der Aufnahme von Sauerstoff und dem der Abgabe von Kohlendioxid. Lavoisier nannte das von Scheele und Priestley entdeckte Gas „oxygene“ (Säurebildner).

\section{Sauerstoff als Medikament \\ $\nabla$}

Sauerstoff als medikamentöse Therapie war im 18. Jahrhundert kaum bekannt und eher mit Mystik und Scharlatanerie verbunden. Berichte von Sauerstoffbehandlungen bei Menschen beschreiben eine belebende und euphorisierende Wirkung. Es wird angenommen, dass herstellungsbedingt Kontaminationen des Atemgases vorgelegen haben. Im anglo-amerikanischen Bereich wurden Atemgasinhalationen über Jahre hinweg als medizinisches „Allheilmittel“ und Wunderdroge propagiert. Indikationen für das Allheilmittel Sauerstoff waren neurologische und psychiatrische Erkrankungen, Hauterkrankungen, generalisierte Infektionen, Pneumonien und Herzerkrankungen. Aufgrund vieler Negativeffekte hat dies der Akzeptanz des Sauerstoffs als

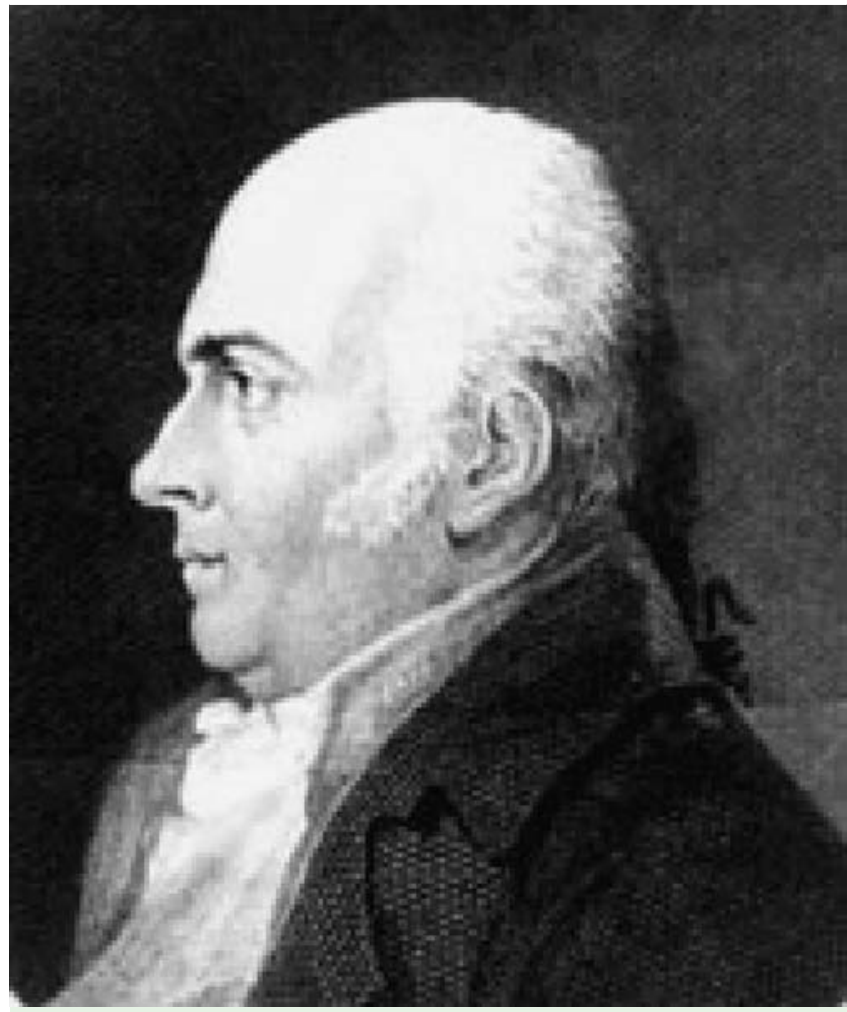

Abb. 4 Thomas Beddoes (1760-1808). Quelle: Wikimedia Commons. 


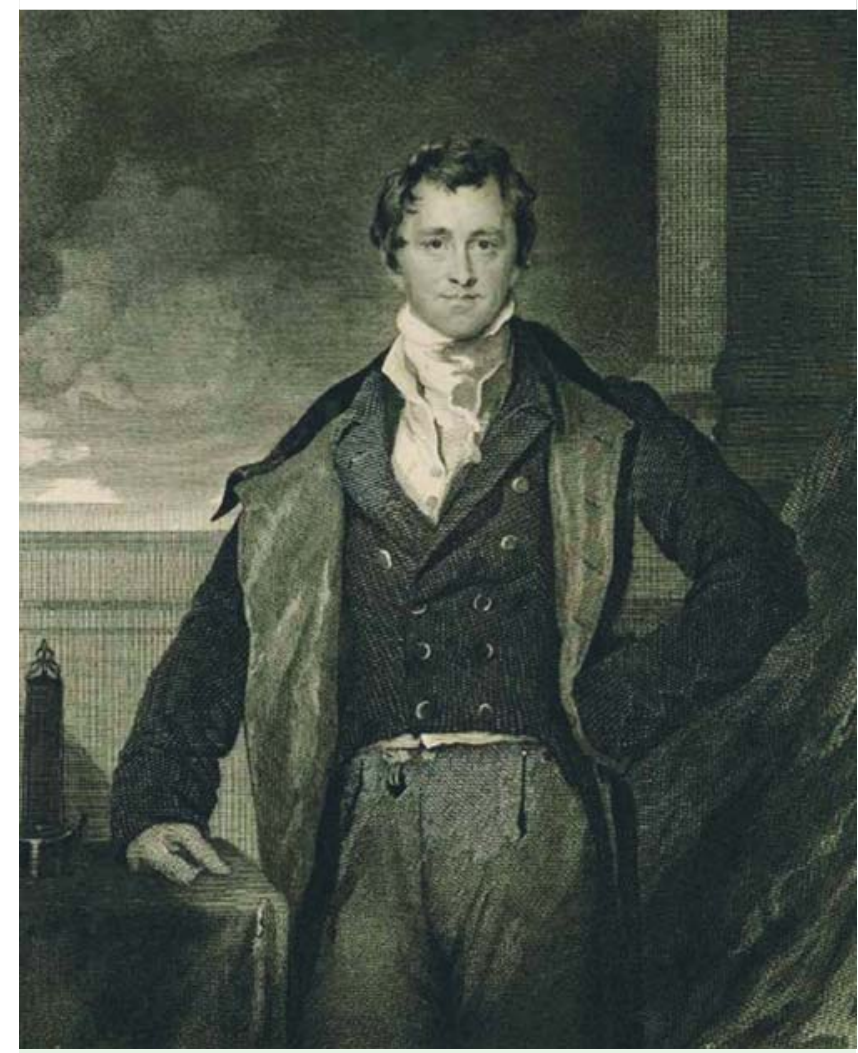

Abb. 5 Humphrey Davy (1778 - 1829). Quelle: Wikimedia Commons.

medizinischem Therapeutikum allerdings eher geschadet als genutzt. Erstmals mit primär therapeutischer Zielsetzung soll Sauerstoff im Jahre 1783 von dem französischen Arzt Caillens angewandt worden sein.

Der englische Arzt und Naturphilosoph Thomas Beddoes (1760 1808) hat im Jahre 1798 in Bristol das erste Institut für Inhalative Gastherapie („Pneumatic Institution for inhalation gas therapy“) aufgebaut. Beddoes und der wissenschaftliche Leiter des Instituts, Humphrey Davy (1778 - 1829), haben in den Folgejahren eifrig mit Gasen, vor allem Lachgas, experimentiert und ihre Daten veröffentlicht. Für die aufwendige Herstellung der Gase war der Maschinenbauer und Entwickler der Dampfmaschine James Watt (1736 - 1819) verantwortlich. Man mutmaßt die Tuberkuloseerkrankung seines Sohnes als agens movens für sein diesbezügliches Engagement. Die Einführung von Sauerstoff als Medikament gelang jedoch nicht, was wahrscheinlich darin begründet lag, dass die applizierten Dosen eher im unterdosierten und damit weitgehend wirkungslosen Bereich gewesen sind. Obwohl das Institut für Gastherapie im Jahre 1802 aufgrund mangelnden Erfolgs wieder geschlossen werden musste, gilt die von Beddoes und Davy begründete „Pneumatische Medizin“ als Ursprung für die Sauerstofftherapie und inhalative Anästhesie.

Abgesehen von einigen kasuistischen Erfolgsberichten über die Anwendung von inhalativem Sauerstoff blieb diese Therapie jedoch umstritten. Bis ins 19. Jahrhundert musste der Sauerstoff von den Anwendern selbst hergestellt werden, zumal eine längerfristige Speicherung nicht möglich war. Die Speicherung von Sauerstoff in Gaszylindern, initial aus Kupfer und später aus Stahl, war erst 1868 nach Entwicklung der Druckgastechnik möglich. Es hat bis ins zweite Jahrzehnt des 20. Jahrhunderts gedauert, bis der Durchbruch bei der klinisch-therapeutischen Anwendung endlich erzielt werden konnte. Neben einem unklaren

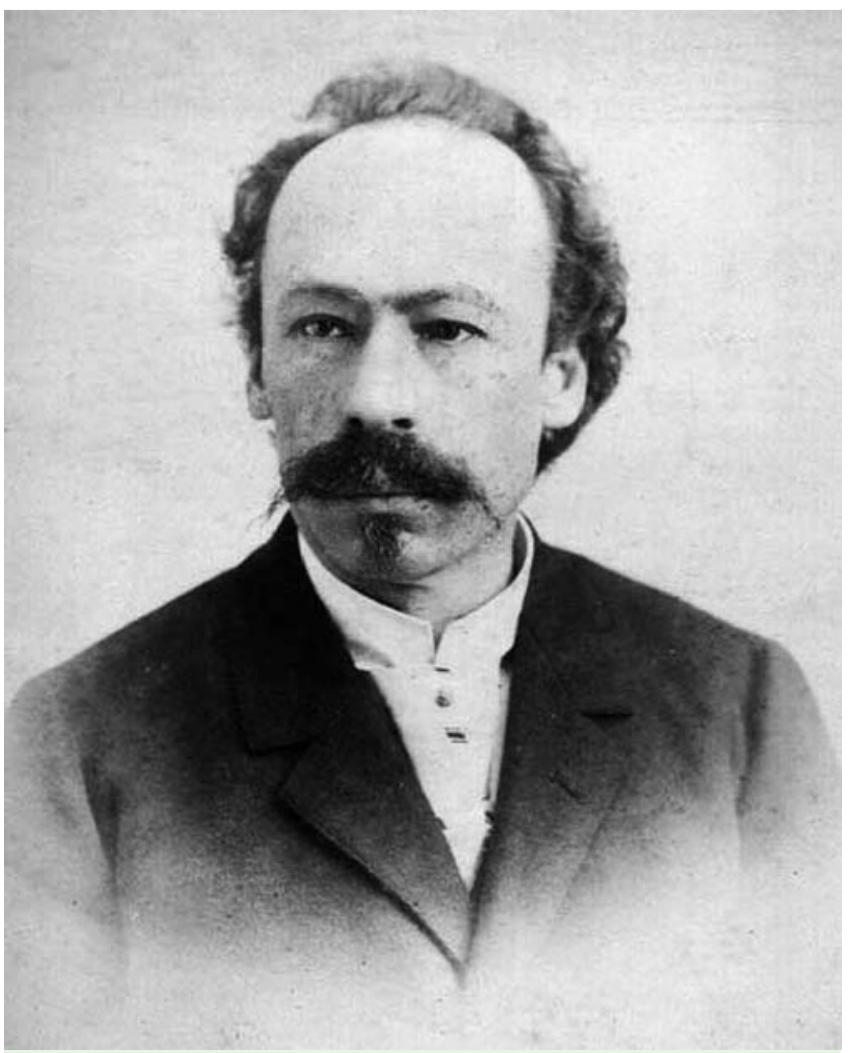

Abb. 6 Karol Stanislaf Olszewski (1846-1915). Quelle: Wikipedia.

physiologischen Verständnis sowie einem nicht eindeutig zu definierenden Indikationsspektrum waren vor allem zwei Faktoren hinderlich: Zum einen die aufwendige und kostenintensive Herstellung des Atemgases, zum anderen die nicht bestehende Möglichkeit einer geregelten dosisgenauen Applikation. Die damalig verfügbaren Applikationsvorrichtungen verfügten über sehr ungenaue Dosierungsvorrichtungen, was verständlicherweise aufgrund des teuer herzustellenden Gases ein Problem darstellte. Neben gasgefüllten Reservoirbeuteln gab es die Möglichkeit die Patienten in Sauerstoff- bzw. Anästhesiekammern zu versorgen. Nach dem ersten Weltkrieg wurden erstmals „Sauerstoffzelte“ etabliert.

\section{Die Verflüssigung von Sauerstoff \\ $\nabla$}

Die Namen Karol Stanislaf Olszewski (1846-1915) und Zygmunt Florenty Wroblewski (1845-1888) sind mit der Verflüssigung des Sauerstoffs untrennbar verbunden. Selbst bekannte Wissenschaftler wie Pierre Eugène Marcelin Berthelot (1827 - 1907), Thomas Andrews (1813 - 1885) und Raoul-Pierre Pictet (1846-1929) scheiterten an dem Versuch, die sogenannten „permanenten“ Gase Sauerstoff, Wasserstoff und Stickstoff zu verflüssigen. Lediglich dem französischen Physiker Louis Paul Cailletet (1832 - 1913) gelang es am 2. Dezember 1877, stark komprimierte Luft mit flüssigem Äthylen bis $-105^{\circ} \mathrm{C}$ abzukühlen und während des Entspannens das Entstehen von Tröpfchen zu erzeugen. Dadurch war zwar offensichtlich geworden, dass sich Luft verflüssigen lässt, der eingeschlagene Weg führte jedoch noch nicht zur Beantwortung der Frage, wie sich dauerhaft Gase als Flüssigkeit stabilisieren ließen. Erst Olszewski, Professor für anorganische Chemie an der Universität Krakau, und dem ebenfalls nach Krakau berufe- 


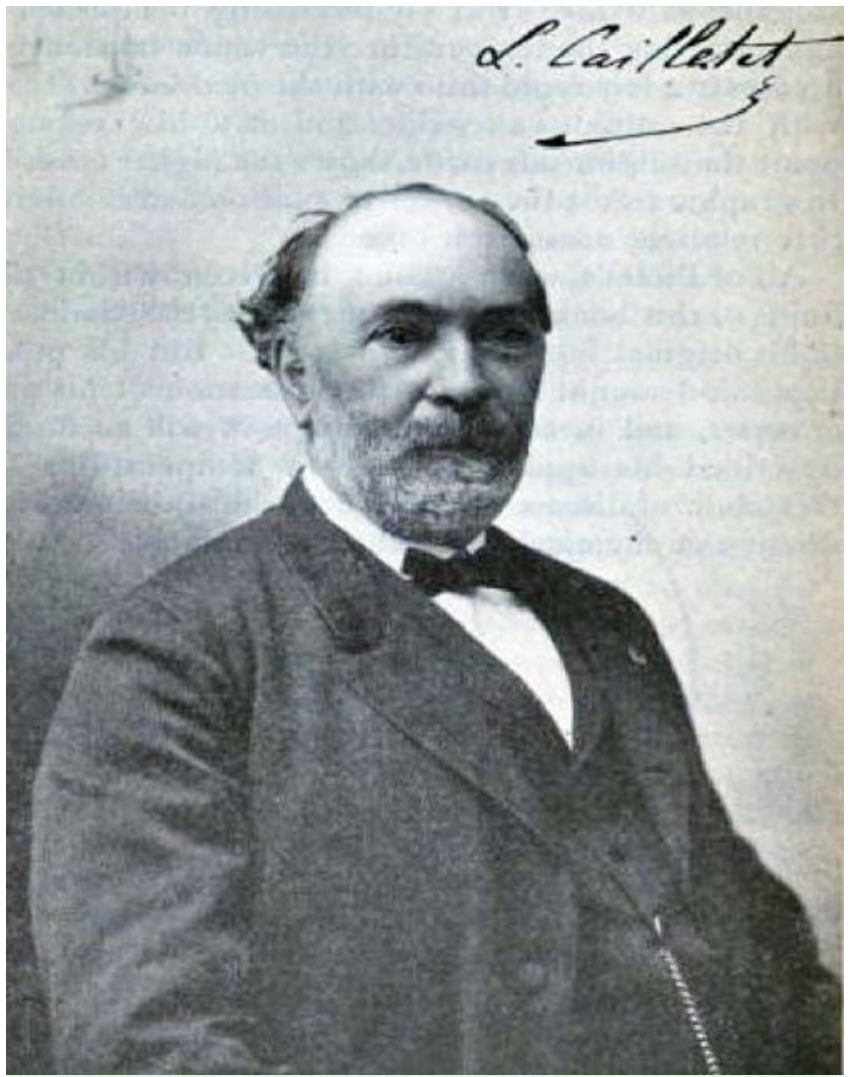

Abb. 8 Louis Paul Cailletet (1832-1913). Quelle: Wikimedia Commons.

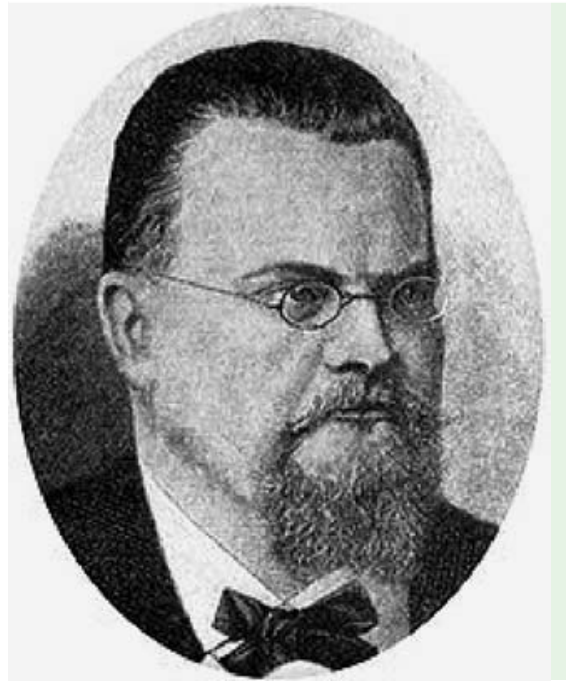

Abb. 7 Zygmunt Florenty Wroblewski (1845-1888). Quelle: Wikipedia.

nen Physiker Wrobleski ist es gelungen, Sauerstoff in Flüssigkeit zu verwandeln und in diesem Zustand zu erhalten. Die beiden Wissenschaftler setzten die Cailletet'schen Versuche fort, indem sie verflüssigtes Äthylen vor dem Ausströmen mit fester Kohlensäure abkühlten. Dadurch erhielten sie eine noch tiefere Temperatur zur Kompression größerer Mengen Sauerstoffs. Olszewski gelang es im Weiteren, unter beständiger Optimierung seiner Apparatur, fast sämtliche damals bekannten Gase zu verflüssigen. Seine Arbeiten bildeten zudem die Basis für die industrielle Anwendung der Kältetechnik.

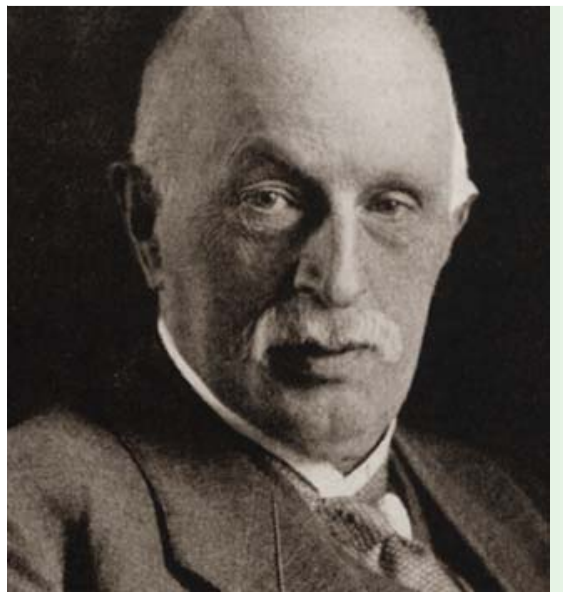

Abb. 9 Heinrich Dräger (1847-1917).

Mit freundlicher Genehmigung von Drägerwerk AG \& Co.KGaA.

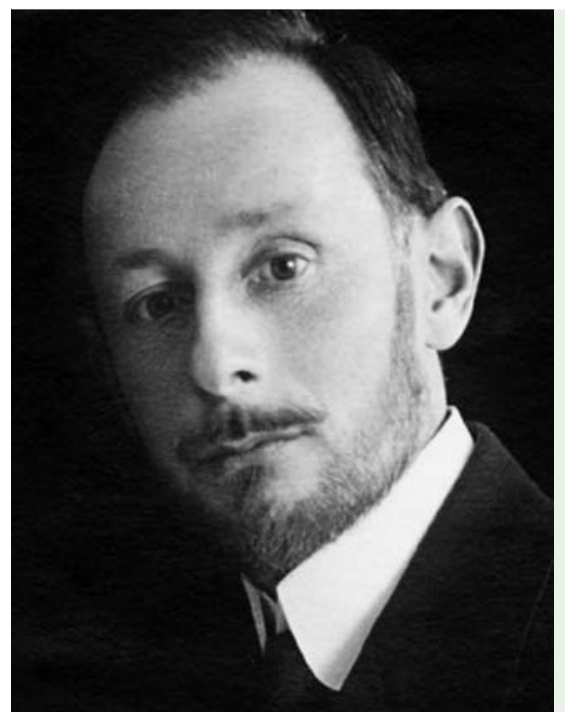

Abb. 10 Dr. Bernhard Dräger (1870-1928). Mit freundlicher Genehmigung von Drägerwerk AG \& Co.KGaA.

\section{Die Druckgas-Ventiltechnologie (Dräger-Technologie) $\nabla$}

Vor allem durch die Entwicklung der Druckgas-Ventiltechnologie durch Heinrich Dräger (1847 - 1917) und Sohn, Dr. Bernhard Dräger (1870-1928), aus Lübeck, wurden die grundlegenden Voraussetzungen geschaffen, verlässlich dosierbare Mengen an Inhalationsgasen zu applizieren. Zwischen den Jahren 1889 und 1902 erfolgte eine intensive Weiterentwicklung der Druckgasventiltechnologie durch die Drägerwerke. Die Präzisionsmechanik der Firma Dräger hat die Beherrschung der Druckgastechnik wesentlich vorangebracht. Die Ventiltechnologie, die bereits im 19. Jahrhundert existierte, hat lange Zeit gebraucht, bis sie sich in der Medizintechnologie etablieren konnte. Dies hat vermutlich seine Erklärung darin, dass die zuvor verfügbaren Ventile sehr schwer (gusseisern, Gewicht etwa $18 \mathrm{~kg}$ ) und zudem noch eingeschränkt funktionsfähig waren. Das erste eigene Druckreduzierventil von Dräger mit Namen „Lubeca“ (1889) war mit 4 kg wesentlich leichter als seine Vorgänger. Die neue Technologie der Druckreduktionsventile hat sich zunächst vor allem in den Bereichen der Anästhesie und Rettungsmedizin durchgesetzt (RothDräger-Narkoseapparat 1902). Sie ermöglichte, das unter hohem Druck gespeicherte Gas mit niedrigerem Druck zurückzuführen, den Gasfluss zu regulieren sowie Gasgemische herzustellen. Eine 

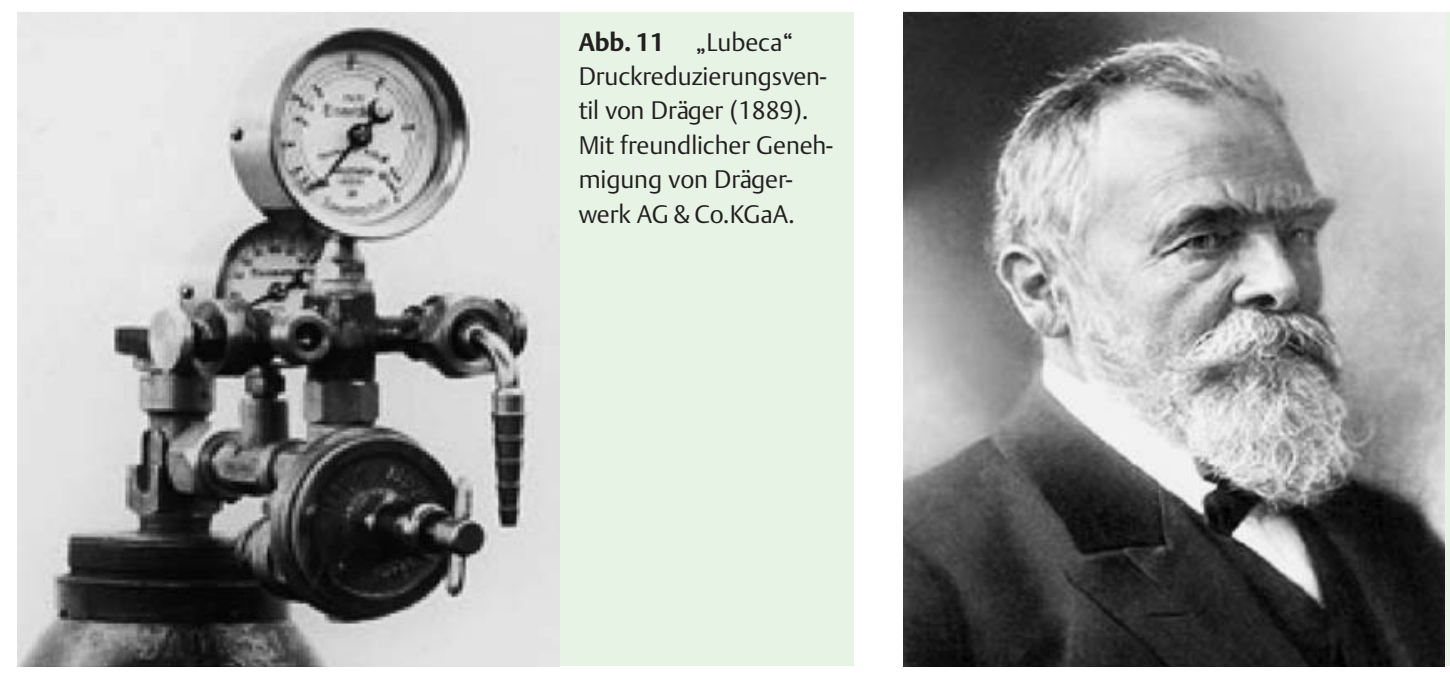

Abb. 13 Carl von Linde (1842-1934).

Mit freundlicher Genehmigung von Linde AG.

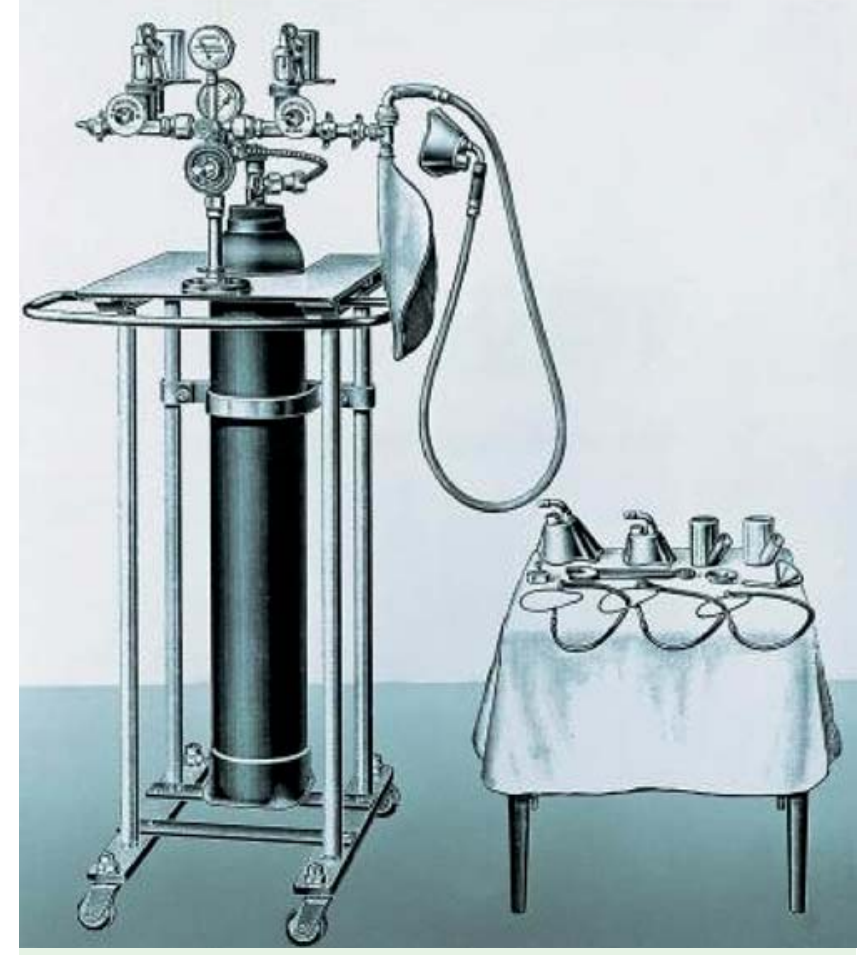

Abb. 12 Roth-Dräger-Narkoseapparat 1902. Mit freundlicher Genehmigung von Drägerwerk AG \& Co.KGaA.

für die industrielle und medizinische Anwendung relevante Bedeutung erlangte Sauerstoff jedoch erst, nachdem ein Weg gefunden worden war, diesen in großen Mengen zu verflüssigen.

\section{Die industrielle Produktion von Sauerstoff ("Linde-Verfahren") \\ $\checkmark$}

Die technische Voraussetzung zur maschinellen Produktion von Sauerstoff war das sogenannte Linde-Verfahren, mit dem Luft verflüssigt und dann in seine Bestandteile zerlegt werden konnte. Das Verfahren hatte der Maschinenbauer und Ingenieur Carl von Linde (1842-1934) bereits 1895 mit der weltersten Versuchsanlage zur Luftverflüssigung etabliert (Patentschrift Nr. 88824; 5. Juni 1895; „Verfahren zur Verflüssigung von Atmo-

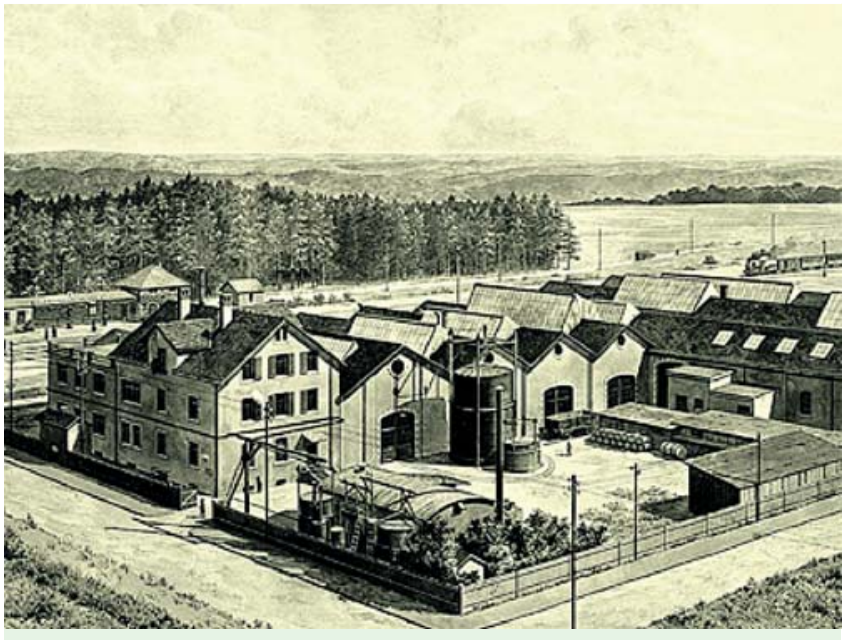

Abb. 14 Die Linde-Werksanlagen in Höllriegelskreuth bei München im Jahre 1909. Mit freundlicher Genehmigung von Linde AG.

sphärenluft bzw. sonstiger Gase“). Beim Linde-Verfahren wird ein Kreislauf erzeugt, in dem mit Hilfe eines Kompressors das Gas verdichtet und gekühlt wird. Anschließend wird das Gas wieder auf atmosphärischen Druck gebracht und wieder in den Kompressor zurückgeleitet. Während des Zurückleitens kühlt es das aus dem Kompressor strömende Gas und durchläuft den Kreislauf von neuem. 1895 konnte mittels des Verfahrens erstmals eine größere Menge flüssige Luft (3 Liter pro Stunde) gewonnen werden. In den Folgejahren wurde die Technologie so weiterentwickelt, dass 1902 die weltweit erste industrielle Luftzerlegungsanlage zur Gewinnung von Sauerstoff in Betrieb genommen werden konnte. Durch das Linde-Verfahren konnte erstmalig industriell und in großem Umfang Flüssigsauerstoff hergestellt werden. Heute können großtechnisch bedeutsame Mengen an gasförmigem und flüssigem Sauerstoff, Stickstoff und Edelgasen mit Hilfe von Luftzerlegungsanlagen hergestellt werden (sog. Fraktionierung). Man nutzt dabei die unterschiedlichen Siedepunkte der einzelnen Luftbestandteile aus. Um diese zu erreichen (Sauerstoff $-183^{\circ} \mathrm{C}$, Stickstoff $-196^{\circ} \mathrm{C}$ ), benutzt man das entspannte Gas im Gegenstromprinzip zur Vorkühlung des verdichteten Gases. 


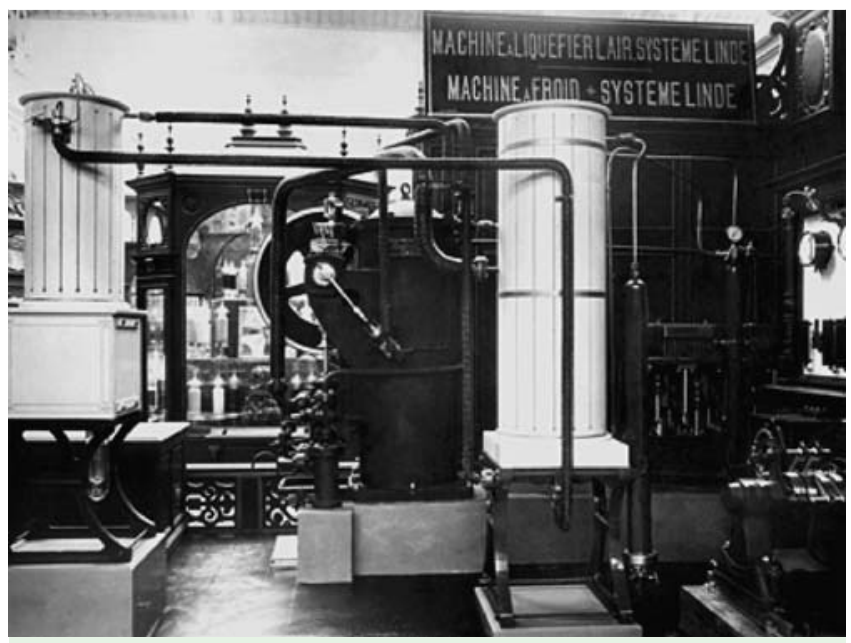

Abb. 15 Linde-Luftverflüssigungsanlage auf der Weltausstellung in Paris im Jahr 1900. Mit freundlicher Genehmigung von Linde AG.

\section{Die „moderne“ Sauerstofftherapie \\ $\nabla$}

Erst mit entsprechenden Technologien und der Gewährleistung der Sicherheit der Applikation hat sich Sauerstoff als Basistherapeutikum in der Medizin durchgesetzt. Mit dem Prinzip der Druckregulierung war der Grundstein einer Technologie gelegt, die es ermöglicht hat, flüssige Gase, insbesondere Sauerstoff, für unterschiedlichste Produkte der Beatmungstechnologie und des Atemschutzes zu nutzen. Die englisch dominierte Geschichtsschreibung hat die Etablierung der modernen Sauerstofftherapie auf das Jahr 1917 festgelegt, vermutlich aus Unkenntnis des deutschen Schriftentums. Vor allem Berichte aus den Manuskripten von John Scott Haldane (1860 - 1936) über die Sauerstoffbehandlung von Giftgasverletzten aus dem 1. Weltkrieg werden im anglo-amerikanischen Ausland als Beginn der modernen Sauerstofftherapie angesehen. Haldanes Forschung hat sicherlich wesentlich zum Verständnis der Atemphysiologie sowie der sinnvollen Anwendung der Sauerstofftherapie beigetragen. Berücksichtigt man aber die Entwicklung der industriellen Herstellung von Sauerstoff sowie die optimierte Applikationsweise mittels Einführung der Reduzierventile, so sollte, entsprechend den Empfeh- lungen von Schmucker et al., das Jahr 1902 als das Jahr der endgültigen Etablierung der modernen Sauerstofftherapie angesehen werden. Es waren erstmals nicht nur die technischen Probleme im Hinblick auf eine dosisgarantierte Applikation des Sauerstoffs gelöst worden, sondern auch diejenigen einer Herstellung des Gases im großindustriellen Maßstab. Somit haben zwei Technologien entscheidend dazu beigetragen, Sauerstoff als medizinisches Basistherapeutikum zu etablieren: zum einen das Verfahren der industriell durchführbaren Luftzerlegung und -verflüssigung, zum anderen das der steuerbaren Applikationsweise mittels Druckreduktionsventilen.

\section{Interessenkonflikt}

$\nabla$

Die Autoren geben an, dass kein Interessenkonflikt besteht.

\section{Literatur}

1 Strätling M, Schmucker P. 100 Jahre Sauerstofftherapie (1902 - 2002) Eine medizinische Neubewertung. Teil I: Der lange Weg von der Entdeckung des Sauerstoffs bis zu seinem Durchbruch bei der therapeutischen Anwendung in der Anästhesiologie und Rettungsmedizin. Anästhesiol Intensivmed Notfallmed Schmerzther 2002; 37: 712 - 720

2 Strätling M, Schmucker P. 100 Jahre Sauerstofftherapie (1902-2002) Eine medizinische Neubewertung. Teil II: Die Bedeutung des RothDräger-Narkoseapparates (1902) und weiterer Entwicklungen aus 100 Jahren Dräger-Medizin- und Anästhesietechnik für die Etablierung der Sauerstoffanwendung im therapeutischen Methodenspektrum. Anästhesiol Intensivmed Notfallmed Schmerzther 2003; 38: 4 13

3 Strätling M, Schmucker P. 100 Jahre Dräger-Medizintechnik (19022002) oder: im Zentrum steht der Sauerstoff... Zur Bedeutung der Druckgastechnik für die Begründung der modernen Sauerstofftherapie und der Anästhesiologie. Anästhesiol Intensivmed Notfallmed Schmerzther 2004; 39 (Suppl. I): 48 - 70

4 Leigh JM. Variation in performance of oxygen therapy devices. Ann Roy Surg Engl 1973; 52: 234-253

5 Birch SB. Action and use of oxygen gas, when administered as a therapeutic agent. The British Medical Journal 1868; 4: 327-329

6 Beneke K. Karol Stanislaw Olszewski und die Geschichte der Verflüssigung von Gasen. Aus: Beneke Klaus. Biographien und wissenschaftliche Lebensläufe von Kolloidwissenschaftlern, deren Lebensdaten mit 1996 in Verbindung stehen. Beiträge zur Geschichte der Kolloidwissenschaften, VII. Mitteilungen der Kolloid-Gesellschaft 1999: 175 - 188

7 Koßobutzki C. Die Geschichte der inhalativen Sauerstofftherapie in Deutschland. Inauguraldissertation medizinische Fakultät der Universität zu Lübeck; 2009 